\title{
Acupuncture point injection with placental extract improves pain and quality of life in patients with fibromyalgia syndrome
}

\author{
Kyeong M Park* and Tae H Cho \\ Cho Orthopaedic \& Oriental Clinic, Seoul 06979, South Korea
}

\begin{abstract}
Context: Fibromyalgia syndrome (FMS) can seriously affect the quality of patient lives due to chronic widespread pain. One of treatment strategies for the management of FMS is complementary and alternative medicine (CAM) treatments including acupuncture. A new acupuncture therapy, acupuncture point injection (API) that combines acupuncture and medication is recently used in China and Korea to accomplish more effective therapeutic outcomes. Yet, there is no attempt to investigate the effect of API with placental extract on the treatment of FMS.
\end{abstract}

Objective: This study evaluated the effectiveness of API with placental extract on pain reduction and life quality in patients with FMS.

Methods: This is a retrospective study analyzing medical records.

Setting: Clinical setting.

Participants: Participants were 64 patients at the clinic being treated for FMS, at an average age of 46 having symptom duration from 6 months to 2 years.

Intervention: Placental extract was weekly injected into acupuncture points GB21, SI11, SI13, SI14, Ex-HN15, and BL13 for five weeks to the participants.

Outcome measures: The primary outcome, pain, was measured on a VAS scale. Secondary outcomes, sleep disorder and daily workable hours without break, were evaluated using questionnaire.

Results: After APIs with placental extract for five weeks, pain and sleep discomfort were substantially decreased. Patients also displayed elongated productive times which denote workable hours to maintain daily activity without pain-evoked break after the API treatment.

Conclusion: We suggest that API with placental extracts is a safe and promising treatment for FMS. A larger follow-up trial is needed to determine more definitely the efficacy of API with placental extract.

\section{Introduction}

Fibromyalgia syndrome (FMS) is a chronic, painful musculoskeletal disorder characterized by widespread pain, pressure hyperalgesia, morning stiffness, sleep disturbances, fatigue, and psychological distress [1]. Diagnosis and therapy may be complex and best outcomes can be achieved with a comprehensive approach [2]. Pharmacological therapies involving anticonvulsants, amitriptyline, and serotonin/ noradrenaline reuptake inhibitors were known to alleviate symptoms of this disease [3].

Patients with FMS utilize complementary and alternative medicine therapies in addition to conventional medicines. Heat application, homeopathy, dietary supplements, acupuncture, and meditative exercises have been used for the management of symptoms in FMS patients [4]. Accumulating evidence suggested that acupuncture is a safe intervention for patients with FMS [5-7]. A systematic review and meta-analysis of randomized controlled trials suggests that the acupuncture is an alternative method of choice in patients with FMS [8].

Acupuncture point injection (API), an injection of diluted drug solution to acupuncture point, is performed to maximize the therapeutic effect of acupuncture in recent days $[9,10]$. A pilot, randomized, double blind, controlled trial demonstrated that API with Carthami Semen decreases chronic daily headache better than control [11]. In adjuvantinduced polyarthritic rats, API with placental extract was shown to alleviate the arthritic symptoms including joint destruction and expression profiles of inflammatory cytokines [12].

Previously we reported that APIs with placental extract successfully relieved pain and restored the articular movement of the affected joints in patients with complex regional pain syndrome [13,14]. Moreover, the APIs with placental extract were demonstrated to be safe for long-term uses $[13,14]$. In this study, we explored whether API with placental extract is a therapeutic choice to decrease pain and ameliorate the quality of life in FMS patients.

${ }^{*}$ Correspondence to: Kyeong Mee Park, Cho Orthopaedic \& Oriental Clinic, Seoul 06979, South Korea, E-mail: kmpark02@empas.com

Key words: fibromyalgia, acupuncture point injection, placental extract, pain, sleep disorder, productive time

Received: May 08, 2020; Accepted: May 15, 2020; Published: May 22, 2020 


\section{Materials and methods}

\section{Subjects}

This retrospective study includes sixty-four patients diagnosed with FMS according to the 1990 American College of Rheumatology classification criteria [15], who presented at Cho Orthopaedic \& Oriental clinic in Seoul, South Korea between May 2014 and December 2015. Patients were in the age range of 15-63 with 9 males and 55 females. Inclusion criteria were widespread pain for 6 months or more, normal neurological examination findings, and having failed to achieve improvement following other complementary and alternative therapies. Exclusion criteria were known bleeding diathesis, autoimmune or inflammatory diseases, pregnancy or lactation, diabetes mellitus, and the use of non-steroidal anti-inflammatory drugs for more than 4 days before the injection. The study was conducted in accordance with the principles of the Declaration of Helsinki. Written informed consent was obtained from each patient.

\section{Placental extract}

Human placental extract obtained under the regulations of the Korean Food and Drug Administration was purchased from Green Cross Wellbeing Ltd. (Yongin, Korea). Briefly, human placentas were collected upon full-term delivery and tested for human immunodeficiency virus and hepatitis $B$ and $C$ viruses. They were then cut into pieces, defatted with acetone, and extracted with water through pepsin and hydrochloric acid-catalyzed hydrolysis. Resulting placental extract was tested for germ-free, anti-histamine, and endotoxin-free under the regulation of Korean Food and Drug Administration. The final placental extract product was sterilized, packaged at $2 \mathrm{ml} /$ ampule, and approved for injection for human. Insoluble macromolecules, such as polysaccharide, polynucleotide, etc. were excluded during the manufacturing processes.

\section{Therapeutic interventions}

Aliquots of placental extract $(2 \mathrm{~mL})$ were weekly injected into the acupuncture points GB21, SI11, SI13, SI14, and Ex-HN15 (Figure 1) of affected side for 5 weeks. In addition, a one $\mathrm{mL}$ aliquot of placental extract was given to acupuncture point BL13 (Figure 1) bilaterally. Sterile $40 \mathrm{~mm}$-long 23-gauge needles were inserted into the acupuncture points.

\section{Data collection and analysis}

Pain score was recorded by the visual analogue scale (VAS) where the score of zero meant that the patients have no pain and the score of 10 is the worst pain. The patients were asked to indicate their feelings of pain by drawing a vertical line on a $10 \mathrm{~cm}$ VAS before and after every week of APIs. The secondary efficacy outcome measures sleep disorder and productive time. Each patient was interviewed for their satisfaction with sleep based on questionnaires comprising whether they have trouble in sleeping, how many hours they slept, and how much they are refreshed after sleep. The indexes were scored from zero to 3 , and high scores indicate severer discomfort of sleep. At the same time, patients were asked to report how many hours they could maintain routine physical activities without fatigue or pain-evoked work break. These routine activities included housework, 30 minute-walking outside, participating in their living communities, and other favorite pursuits and were scored from zero to 12 . The results were expressed as the productive time scores, in which low scores indicate more severe disability. All data are expressed as the median with interquartile range (IQR).

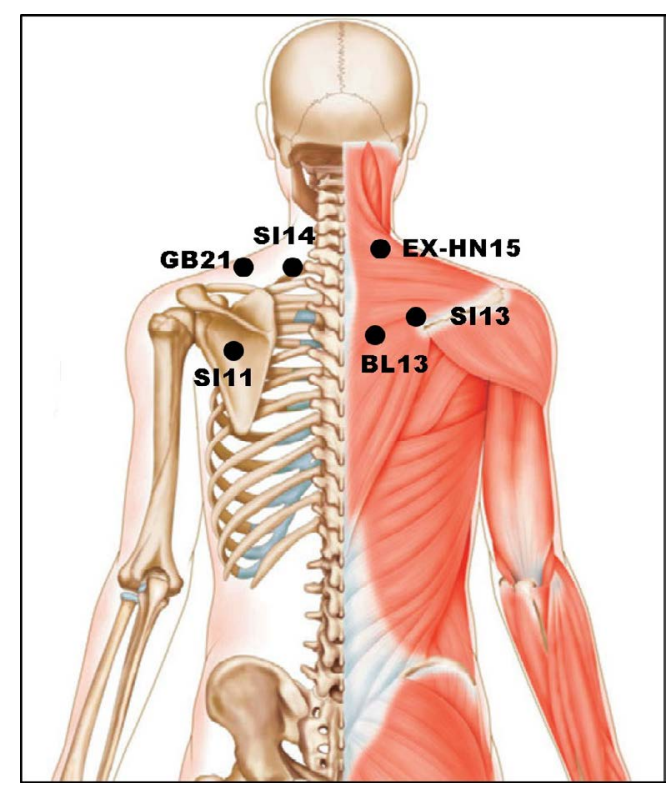

Figure 1. Loci of acupuncture points used in the treatment of FM.

Data were analyzed using SPSS (SPSS Inc., Chicago, IL, USA, Version 18.0). To evaluate the changes of VAS, sleep disorder, and productive time by successive APIs in FM patients, the non-parametric test of Wilcoxon signed rank was applied; the assessment of distributions was made using the Shapiro-Wilk test. Statistical significance was set at $\mathrm{P}<0.01$.

\section{Results}

Patients with FMS expressed a wide range of pain as expressed by VAS 3 to VAS 7 before API with placental extract. The value of VAS was gradually decreased by successive APIs with placental extract and finally reached to the median value of 1 (Figure 2). Additionally, improvement of quality of life was also achieved by successive APIs with placental extract. At the start of API with placental extract, the index of patients' sleep disorder displayed the median value of 2 (Figure 3). The index was gradually decreased with the successive APIs with placental extract and reached to the median value of 0 (Figure 3). For daily working hours without break, productive time was ranged from 2 to 9 with the median value of 6 before API with placental extract (Figure 4). After the fifth API with placental extract, the median value of productive time increased to 9 (Figure 4).

\section{Discussion}

FMS is a chronic musculoskeletal pain condition whose etiology is inconclusive. Nociceptive stimuli from muscle tissues are recognized as being relevant to the development of FMS $[16,17]$. Local pain of FMS patients is often related to the presence of myofascial trigger points (MTPs). Simons et al. [18] suggested that MTPs are an important factor in the genesis of FMS.

FMS patients show higher pain sensitivity and lower pressure pain thresholds over the cervical spine and the upper back muscle [19]. The outcome of cervical pathology verified that the MTPs are commonly observed in the neck, parascapular region, and upper back muscles [20]. We take the acupuncture points located on the MTPs of the upper back muscle and the paraspinal muscle for treating FMS patients in this study. Ex-HN15 is localized on the paraspinal muscle between C6 and C7 while SI13, BL13, GB21, SI11, and SI14 coincide with the MTPs of the supraspinatus, upper rhomboid major, upper trapezius, infraspinatus, 


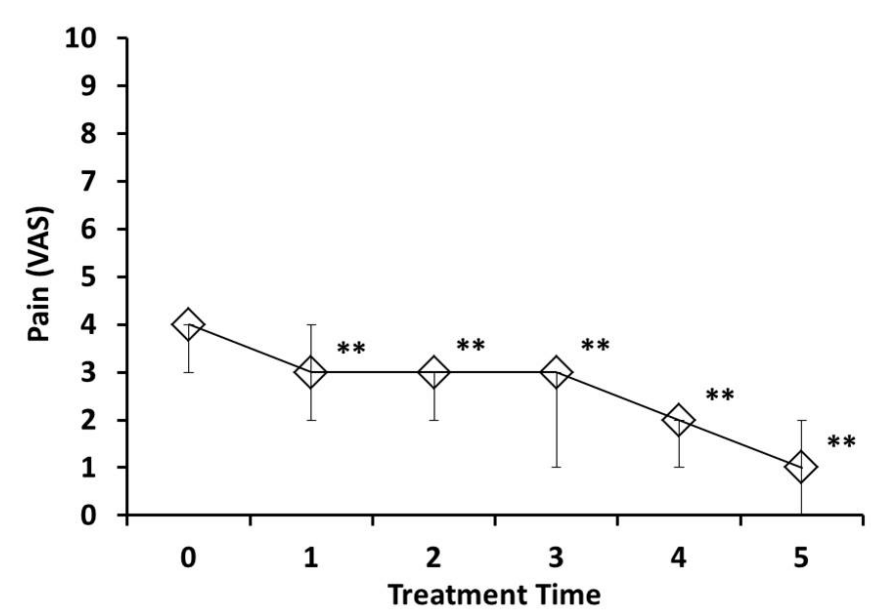

Figure 2. Effects of API with placental extract on pain. The severity of pain was expressed by VAS. The median with IQR, in which error bars show the 1st and the 3rd quartiles was shown. Not displayed upper or lower error limit is overlapped with the median. ** denotes a statistical significance with $\mathrm{p}<0.01$, compared with the untreated control.

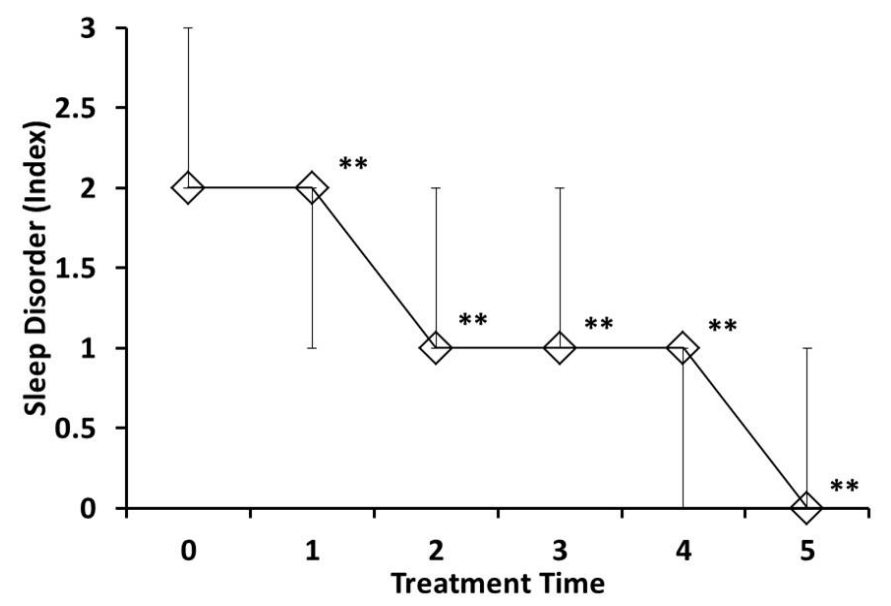

Figure 3. Effects of API with placental extract on sleep disorder. Values on the Y-axis represent degrees of sleeping discomfort derived by questionnaire surveys. The median with IQR, in which error bars show the 1st and the 3rd quartiles was shown. ** denotes a statistical significance with $\mathrm{p}<0.01$, compared with the untreated control.

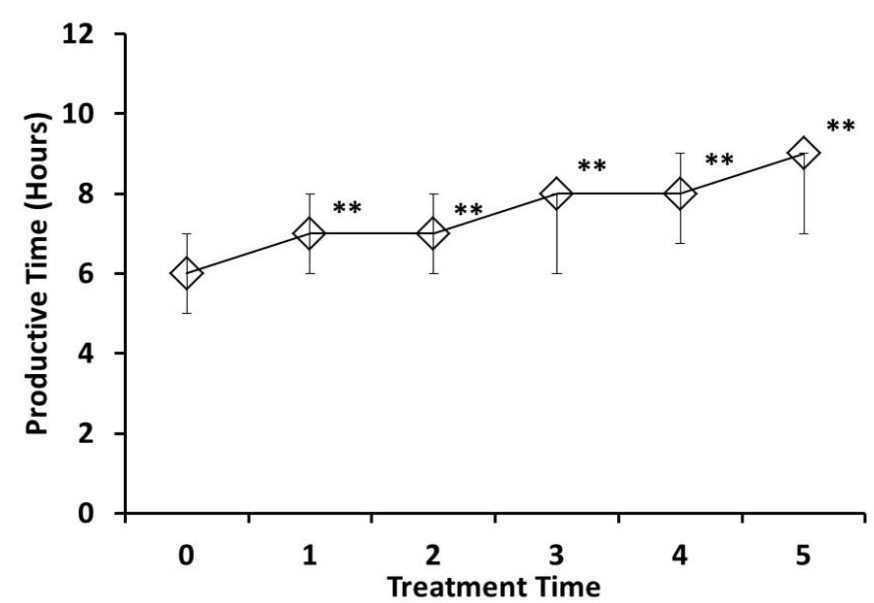

Figure 4. Effects of API with placental extract on productive time. Values of Y-axis represent the daily hours of routine work without a break due to pain. The median with IQR, in which error bars show the 1 st and the 3 rd quartiles was shown. ${ }^{* *}$ denotes a statistical significance with $\mathrm{p}<0.01$, compared with the untreated control. and levator scapular muscles, respectively. APIs with placental extract to GB21, SI11, SI13, SI14, Ex-HN15, and BL13 improved the symptoms of FMS by relieving complications derived from the pathological condition of neck. The inhibition of MTPs by stimulation of these acupuncture points might decrease their nociceptive input, normalize the synaptic efficacy, and reduce peripheral and central sensitization. In particular, APIs to Ex-HN15 and BL13 which have rich nociceptive receptors $[21,22]$ could activate sensitized polymodal type receptors and relieve the fatigue of the cervical extensor muscles, resulting in pain relief.

In fact, API is derived from intramuscular injection in Western medicine and integrated into traditional Chinese medicine [23]. The medical agents administered in acupuncture points are thought to play a synergistic effect with acupuncture point stimulation, and this method is believed to have a more sustained effect than the traditional acupuncture needling or simple intramuscular injection [24]. We speculate that anti-inflammation [25,26], anti-insomnia [27,28], and anti-fatigue $[29,30]$ of placental extract might afford a sound basis for the reduction of pain, decrease of sleep disorder, and increase of productive time in this study. Increase of productive time which covers spending times for daily physical activities without sensation of fatigue and pain-evoked rest implies that patients with FMS manage their daily life more productively after successive APIs with placental extract.

Though the effects of API have been widely demonstrated [31-33], little information is available in its therapeutic mechanism of action. Chen et al. suggested that both the spatial configuration changes and liquid substrate stimulate the acupuncture point and activate neuronal signal transmission system [34]. Moreover, Chao et al. demonstrated that the plasma concentration of injected drug changed significantly after API [35]. This study premised that the distension by placental extract injection at acupuncture points could serve as a local volume reservoir from which the supposed therapeutic factors in placental extract could be subsequently absorbed into the local blood vessels.

Our study limitations include the lack of a placebo control group, not being blind, and sex difference. Studies that include larger sample size are needed to validate the findings from our study. The mechanism through which injecting placental extract at acupuncture points successfully modulates the symptoms of FMS demands future studies. Whether the combined treatment with acupuncture and placental extract may evoke synergistic effects is the subject of our future studies.

\section{Conflict of Interests}

The authors declare that there is no conflict of interests regarding the publication of this paper. This study was carried out without any kind of governmental or academic funds.

\section{Acknowledgment}

We appreciate Dr. Cheol O Joe, Korea Advance Institute of Science \& Technology for comments on this manuscript.

\section{References}

1. Hauser W, Hayo S, Biewer W, Gesmann M, Kuhn-Becker H, et al. (2010) Diagnosis of fibromyalgia syndrome-a comparison of Association of the Medical Scientific Societies in Germany, survey, and American College of Rheumatology criteria. Clin J Pain 26: 505-511. [Crossref]

2. Imamura M, Cassius DA, Fregni F (2009) Fibromyalgia: From treatment to rehabilitation. Eur J Pain 3: 117-122. [Crossref]

3. Clauw DJ (2008) Pharmacotherapy of patients with fibromyalgia. J Clin Psychiatry 69: 25-30. [Crossref] 
4. Hauser W, Jung E, Erbsloh-Moller B, Gesmann M, Kuhn-Becker H, et al. (2012) German fibromyalgia consumer reports. Benefits and harms of fibromyalgia syndrome therapies. Schmerz 26: 150-159. [Crossref]

5. Wolfe F, Clauw DJ, Fitzcharles MA, Goldenberg DL, Katz RS, et al. (2010) The American College of Rheumatology preliminary diagnostic criteria for fibromyalgia and measurement of symptom severity. Arthritis Care Res (Hoboken) 62: 600-610. [Crossref]

6. Wolfe F, Ross K, Anderson J, Russell IJ, Hebert L (1995) The prevalence and characteristics of fibromyalgia in the general population. Arthritis Rheum 38: 19-28. [Crossref]

7. Alarcon GS, Bradley LA (1998) Advances in the treatment of fibromyalgia: current status and future directions. Am J Med Sci 315: 397-404. [Crossref]

8. Zhang XC, Chen H, Xu WT, Song YY, Gu YH, et al. (2019) Acupuncture therapy for fibromyalgia: a systematic review and meta-analysis of randomized controlled trials. $J$ Pain Res 12: 527-542. [Crossref]

9. Yeom MJ, Lee HC, Kim GH, Lee HJ, Shim I, et al. (2006) Anti-arthritic effects of Ephedra sinica STAPF herb-acupuncture: inhibition of lipopolysaccharide-induced inflammation and adjuvant-induced polyarthritis. J Pharmacol Sci 100: 41-50. [Crossref]

10. Marx C, Silveira MD, Selbach I, da Silva AS, Braga LM, et al. (2014) Acupoint injection of autologous stromal vascular fraction and allogeneic adipose-derived stem cells to treat hip dysplasia in dogs. Stem Cells Int 2014: 391274. [Crossref]

11. Park JM, Park SU, Jung WS, Moon SK (2011) Carthami-Semen acupuncture point injection for chronic daily headache: a pilot, randomised, double-blind, controlled trial. Complement Ther Med 1: S19-25. [Crossref]

12. Yeom MJ, Lee HC, Kim GH, Shim I, Lee HJ, et al. (2003) Therapeutic effect of Hominis placenta injection into an acupuncture point on the inflammatory responses in subchondral bone region of adjuvant-induced polyarthritic rat. Biol Pharm Bull 26: 1472-1477. [Crossref]

13. Cho TH, Park KM (2014) Complex regional pain syndrome type 1 relieved by acupuncture point injections with placental extract. J Acupunct Meridian Stud 7: 155158. [Crossref]

14. Cho TH, Park KM (2016) Use of Acupuncture point injection with placental extract for treatment of complex regional pain syndrome. J Pain Relief 5: 246.

15. Wolfe F, Smythe HA, Yunus MB, Bennett RM, Bombardier C, et al. (1990) The American college of rheumatology 1990 criteria for the classification of fibromyalgia Report of the multicenter criteria committee. Arthritis Rheum 33: 160-172. [Crossref]

16. Vierck CJ Jr (2006) Mechanisms underlying development of spatially distributed chronic pain (fibromyalgia). Pain 124: 242-263. [Crossref]

17. Alonso-Blanco C, Fernandez-de-las- Penas C, Morales-Cabezas M, Zarco- Moreno P, Ge HY, Florez-Garcia M (2011) Multiple active myofascial trigger points reproduce the overall spontaneous pain pattern in women with fibromyalgia and are related to widespread mechanical hypersensitivity. Clin J Pain 27: 405-413. [Crossref]

18. Simons DG, Travell J, Simons LS (1999) Myofascial Pain and Dysfunction: The Trigger Point Manual. Williams \& Wilkins, Baltimore.

19. Castro-Sanchez AM, Mataran-Penarrocha GA, Lopez-Rodriguez MM, Lara-Palomo IC, Arendt-Nielsen L, et al. (2012) Gender differences in pain severity, disability, depression, and widespread pressure pain sensitivity in patients with fibromyalgia syndrome without comorbid conditions. Pain Med 13: 1639-1647. [Crossref]
20. Sari H, Akarirmak U, Uludag M (2012) Active myofascial trigger points might be more frequent in patients with cervical radiculopathy. Eur J Phys Rehabil Med 48: 237-244. [Crossref]

21. Takahashi Y, Ohtori S, Takahashi K (2010) Dorsoventral organization of sensory nerves in the lumbar spine as indicated by double labeling of dorsal root ganglion neurons. J Orthop Sci 15: 578-583. [Crossref]

22. Ashton IK, Ashton BA, Gibson SJ, Polak JM, Jaffray DC, et al. (1992) Morphologica basis for back pain: the demonstration of nerve fibers and neuropeptides in the lumbar facet joint capsule but not in ligamentum flavum. J Orthop Res 10: 72-78. [Crossref]

23. Wang M, Gao YH, Xu J, Chi Y, Wei XB, et al. (2015) Zusanli (ST36) acupoint injection for preventing postoperative ileus: A systematic review and meta-analysis of randomized clinical trials. Complement Ther Med 23: 469-483. [Crossref]

24. Zhu YH, Chen YH (2005) On effects of acupoints and drugs in acupoint-injection treatment. Zhongguo Zhen Jiu 25: 46-48. [Crossref]

25. Liu J, Luo S, Yang J, Ren F, Zhao Y, et al. (2018) The Protective Effect of Sheep Placental Extract on Concanavalin A-induced Liver Injury in Mice. Molecules 24: 28. [Crossref]

26. Kim BY, Park HR, Shin JH, Kim SW, Kim SW (2014) Human placental extract reduces allergic inflammation in a murine allergic rhinitis model. Laryngoscope 124 E399-404. [Crossref]

27. Koike K, Yamamoto Y, Suzuki N, Yamazaki R, Yoshikawa C, et al. (2013) Efficacy of porcine placental extract on climacteric symptoms in peri- and postmenopausal women. Climacteric 16: 28-35. [Crossref]

28. Gurgel LA, Santos FA, Rao VS (2000) Effects of human placental extract on chemical and thermal nociception in mice. Eur J Pain 4: 403-408.

29. Lee KK, Choi WS, Yum KS, Song SW, Ock SM, et al. (2012) Efficacy and safety of human placental extract solution on fatigue: a double-blind, randomized, placebocontrolled study. Evid Based Complement Alternat Med 2012: 130875. [Crossref]

30. Park SB, Kim KN, Sung E, Lee SY, Shin HC (2016) Human Placental Extract as Subcutaneous Injection Is Effective in Chronic Fatigue Syndrome: A Multi-Center, Double-Blind, Randomized, Placebo-Controlled Study. Biol Pharm Bull 39: 674-679. [Crossref]

31. Kang SY, Kim CY, Roh DH, Yoon SY, Park JH, et al (2011) Chemical stimulation of the ST36 acupoint reduces both formalin-induced nociceptive behaviors and spinal astrocyte activation via spinal alpha-2 adrenoceptors. Brain Res Bull 86: 412-421. [Crossref]

32. Choi HS, Kang SY, Roh DH, Choi SR, Ryu Y, et al (2018) Bee venom stimulation of a lung meridian acupoint reduces inflammation in carrageenan-induced pleurisy: an alternative therapeutic approach for respiratory inflammation. J Vet Sci 19: 708-715. [Crossref]

33. Kim JK, Kim TH, Park SW, Kim HY, Kim SH, et al (2010) Protective effects of human placenta extract on cartilage degradation in experimental osteoarthritis. Biol Pharm Bull 33: 1004-1010. [Crossref]

34. Chen CY, Lin CN, Chern RS, Tsai YC, Chang YH, et al. (2014) Neuronal activity stimulated by liquid substrates injection at Zusanli (ST36) acupoint: The possible mechanism of aquapuncture. Evid Based Complement Alternat Med 2014: 627342. [Crossref]

35. Chao MT, Wade CM, Booth SL (2014) Increase in plasma phylloquinone concentrations following acupoint injection for the treatment of primary dysmenorrhea. $J$ Acupunct Meridian Stud 7: 151-154. [Crossref]

Copyright: (C2020 Park KM. This is an open-access article distributed under the terms of the Creative Commons Attribution License, which permits unrestricted use, distribution, and reproduction in any medium, provided the original author and source are credited. 